CARNETS OE Carnets de géographes

GÉOGRAPHES.

9 | 2016

Géographies des émotions

\title{
Appréhender les émotions dans le contexte d'une thèse CIFRE
}

Tentative d'objectivation face à la crise paysagère du canal du Midi

\section{Lisa Rebolledo}

\section{OpenEdition}

\section{Journals}

Édition électronique

URL : http://journals.openedition.org/cdg/657

DOI : $10.4000 /$ cdg. 657

ISSN : 2107-7266

\section{Éditeur}

UMR 245 - CESSMA

\section{Référence électronique}

Lisa Rebolledo, "Appréhender les émotions dans le contexte d'une thèse CIFRE », Carnets de géographes [En ligne], 9 | 2016, mis en ligne le 30 novembre 2016, consulté le 01 mai 2019. URL: http://journals.openedition.org/cdg/657 ; DOI : 10.4000/cdg.657

\section{(c)}

La revue Carnets de géographes est mise à disposition selon les termes de la Licence Creative Commons Attribution - Pas d'Utilisation Commerciale - Pas de Modification 4.0 International. 


\title{
Appréhender les émotions dans le contexte d'une thèse CIFRE Tentative d'objectivation face à la crise paysagère du canal du Midi
}

\author{
Lisa REBOLLEDO \\ Doctorante en géographie, \\ Université Paris 1 Panthéon-Sorbonne, EIREST \\ lyssa.rebolledo@gmail.com
}

\begin{abstract}
Résumé
Dans le cadre d'une thèse bénéficiant d'un contrat CIFRE avec le gestionnaire de mon objet d'étude, le canal du Midi, j'ai pu assister quotidiennement à l'expression de diverses formes d'émotions, allant de l'admiration pour son concepteur à l'indignation concernant son entretien, en passant par la dépression face à une gestion de projet conflictuelle (pouvant mener à des changements organisationnels). Travaillant sur le jeu des acteurs et enjeux de gouvernance dans un contexte de bouleversement paysager de ce site inscrit sur la liste du patrimoine mondial, bouleversement perçu comme une véritable " catastrophe ", j'ai pu recueillir le point de vue d'acteurs territorialisés aux objectifs parfois divergents, ou du moins qui leur semblent l'être : maintien du bon fonctionnement de l'ouvrage hydraulique, exploitation économique et touristique, préservation du patrimoine, maintien de la biodiversité, etc. Ces diverses conceptions m'ont naturellement menée à m'interroger sur les représentations et les valeurs qui sont attachées à ce site et me paraissant pouvoir être corrélées à des émotions. En ce sens, l'émotion serait la preuve du patrimoine. Cet article a donc pour objectif d'expliciter une démarche réflexive sur mon positionnement face à des émotions négatives, de prendre en compte d'autres émotions qui m'affectent moins et, enfin, de dépasser cette appréhension pour nourrir le contenu de ma thèse et voir les émotions comme une lecture possible des liens complexes entre acteurs.
\end{abstract}

Mots-clefs : émotions, recherche de terrain, Canal du Midi, paysage, patrimoine.

\begin{abstract}
Employed by the managing organization of Canal du Midi, which is the object of my thesis, I witnessed daily the expression of various forms of emotions, from admiration for its designer, to indignation concerning its maintenance, through depression in front of a controversial project. Working on the actors and governance issues, in the context of a landscape upheaval deemed as a real "disaster" for this Unesco World Heritage Site, I collected several points of view from territorialized actors sometimes persuings opposite objectives: the proper
\end{abstract}


functioning of the hydraulic structure, economic and tourism development, heritage conservation, biodiversity maintenance, etc. These various conceptions led me to wonder about the representations and values attached to this site and seemed to me to be correlated with emotions. Indeed, emotion could be the proof of heritage. Therefore, this article aims at clarifying a reflective process on my own situation in front of negatives emotions, at considering other emotions that affect me less, and finally at overcoming this apprehension to enrich the content of my thesis and conceive emotions as a way to better understand the complex relationships between actors.

Keywords: emotions, field research, Canal du Midi, landscape, heritage. 


\section{Introduction}

Malaise en réunion. Des larmes coulent en face de moi ; à ma droite, un mécanisme de défense, de soutien et de détournement de l'attention s'est enclenché. Mes yeux ne savent plus où regarder, le rouge sur d'autres joues démontre une humeur difficilement contenue, probablement mêlée de colère et de larmes retenues. Pour le reste de l'assemblée, c'est un sentiment de gêne qui nous envahit. Intérieurement, je me demande ce que je fais là. En contrat CIFRE (Convention industrielle de formation par la recherche) avec le gestionnaire de mon terrain d'étude, le canal du Midi, entendre des accès de colère résonner dans les couloirs n'était plus surprenant. Néanmoins, assister à une telle détresse exprimée en présence de personnes extérieures à la structure, lors d'une réunion qui me paraissait importante pour l'avancée de ma thèse, m'a, sur le moment, montré toute l'ambiguïté de mon positionnement d'apprentie-chercheuse : comment rendre compte de ce quotidien, qui n'est pas fondamental pour ma démonstration, mais qui affecte ma façon de penser, de travailler et d'appréhender mes recherches, alors que les relations entre les différents acteurs y sont centrales ? Comment parvenir à prendre du recul ? Dans ce contexte, j'ai considéré l'appel à contributions pour ce neuvième numéro des Carnets de géographes comme une opportunité pour m'aider à saisir et à expliciter mon positionnement. II m'est alors apparu qu'au-delà de mon appréhension face à ces émotions, uniquement négatives, il importait de prendre en compte des émotions plus positives, et qu'il était aussi possible d'utiliser certaines d'entre elles comme clé de lecture d'actions sociales pouvant impacter des faits spatiaux sur mon terrain d'étude.

Ce dernier (Figure 1) présente des caractéristiques physiques et administratives qui démultiplient les approches scientifiques possibles. Propriété de l'État, sa gestion est confiée à Voies Navigables de France (VNF) en 1991, un Établissement public administratif (ÉPA) depuis 2013. Reliant Toulouse à Sète, il traverse deux régions (Midi-Pyrénées et LanguedocRoussillon, qui n'en font plus qu'une depuis la fin de l'année 2015, la région Occitanie / Pyrenées-Méditerranée), trois départements ${ }^{1}$ (quatre si on inclut le système d'alimentation en eau en montagne Noire). II fait aussi partie d'un ensemble plus vaste également géré par VNF, le canal des Deux Mers, avec le canal latéral à la Garonne, qui relie Toulouse à Castetsen-Dorthe (Aquitaine, Gironde). Le canal du Midi, la partie la plus ancienne de cet ensemble, représente un linéaire de $240 \mathrm{~km}$, qui traverse une métropole, des villes, des villages, des espaces ruraux et péri-urbains. Inscrit sur la liste du patrimoine mondial en 1996 comme bien culturel, il a été classé au titre de la loi de 1930, tandis que plusieurs de ses ouvrages d'art sont classés ou inscrits au titre des Monuments historiques. Enfin, cet ouvrage hydraulique a perdu sa fonction d'origine (transport de marchandises) et a été défini comme faisant partie du réseau à vocation touristique tout en continuant à irriguer certaines terres agricoles.

\footnotetext{
${ }^{1}$ La Haute-Garonne, l'Aude et l'Hérault.
} 
Figure 1 : Le réseau des voies navigables du Sud-Ouest

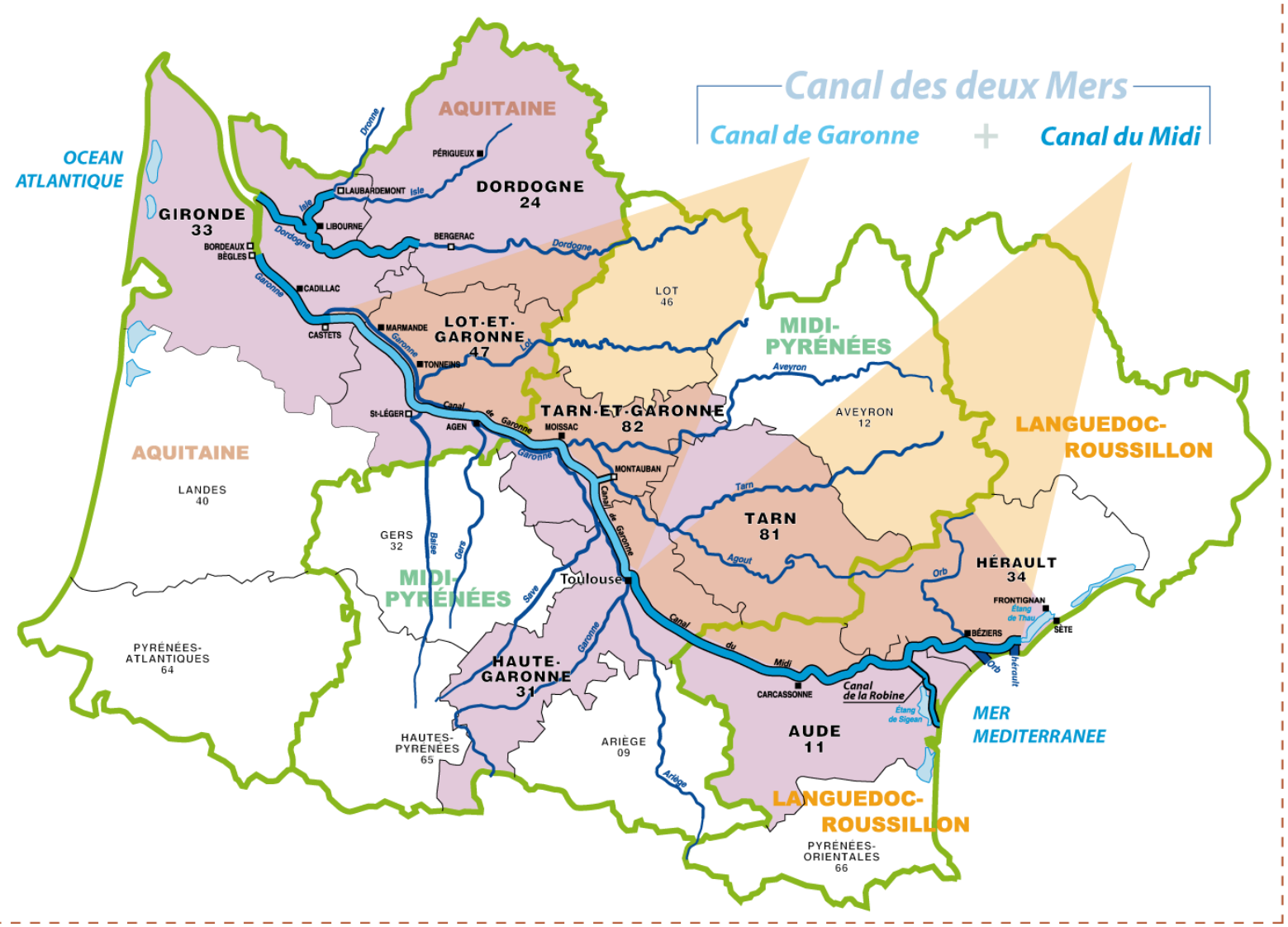

Source : VNF, 5 septembre 2008, en ligne : http://www.sudouest.vnf.fr/reseau-des-voies-navigables-du-sudouest-r138.html

Actuellement, ce site connaît un bouleversement paysager très important, avec la disparition de ses alignements de platanes, atteints par la maladie du chancre coloré qui entraîne un dépérissement en deux à trois ans et oblige son gestionnaire à effectuer un abattage massif, aucun traitement n'ayant pu être trouvé à ce jour. La restauration du canal du Midi, qui a fait l'objet d'une longue réflexion (sous la forme d'un Cahier de référence), validée par la Commission supérieure des sites, perspectives et paysages (CSSPP) en 2012, a été élaborée en suivant cinq recommandations émanant du ministère de l'Écologie: " grandeur à termes des essences choisies restituant l'effet de colonnades et de voûte arborée, espacement faible entre les arbres, la symétrie des rives, l'homogénéité des essences sur de grands tronçons, transparence aux écluses " (VNF, 2015). Selon ces préconisations, le projet végétal se constitue autour d'une essence jalon, récemment définie, comme le chêne chevelu que l'on retrouvera sur $40 \%$ du linéaire, et d'essences intercalaires telles que le micocoulier, le peuplier blanc ou l'orme résistant. II ne s'agit donc pas d'une restauration à l'identique, mais d'un projet qui tente de concilier valeurs patrimoniales (avec le choix de retrouver un aspect global hérité des plantations du XVIII et $X I X{ }^{e}$ siècles) et obligations environnementales puisqu'il s'agit d'éviter une nouvelle pandémie favorisée par la généralisation d'une essence unique.

Partant du constat que ce phénomène mettait en exergue les dysfonctionnements en termes de gouvernance d'un site qui implique des acteurs se situant à des échelles différentes, ma problématique s'est construite à partir de ce point de rupture, qui a pu être 
qualifié de "traumatisant". II s'agit de savoir si ce point de rupture implique un repositionnement des différents acteurs territorialisés (Gumuchian et al., 2003) et quels impacts il pourrait avoir sur les représentations et pratiques des touristes. Philippe Violier propose d'analyser les jeux des acteurs en deux étapes: une approche taxonomique, qui consiste à dresser un inventaire des personnes ou groupes de personnes intervenant dans le monde du tourisme au niveau global ou local, puis une approche systémique, qui analyse les relations entre ces acteurs et avec "le territoire dans lequel leur action s'inscrit " (2003: 174). Cette double approche pour catégoriser ces acteurs sert ici de point de départ, pour recueillir le point de vue d'acteurs relevant de ces différentes catégories et représentant diverses échelles territoriales. Des entretiens semi-directifs ont été menés avec des acteurs entrepreneurs, dans laquelle sont ici inclus les loueurs de bateaux, les propriétaires de bateaux-promenades, de péniches hôtels, etc. Les acteurs publics représentent une catégorie très importante et se partagent entre les collectivités territoriales et l'État. Pour les premiers, j'ai pu m'entretenir avec des élus et des techniciens (communes, intercommunalités, départements, régions). Du côté de l'État, j'ai régulièrement pu recueillir le point de vue des agents des services déconcentrés (DREAL, DRAC, etc.) et de l'administration centrale (ministère de l'Environnement, ministère de la Culture), soit lors d'entretiens bilatéraux, soit lors de réunions diverses. Je suis enfin allée à la rencontre des touristes (fluviaux ou fluvestres) et des habitants, en effectuant de courts entretiens, des échanges informels ou de l'observation. L'ensemble de ma méthodologie repose donc sur des entretiens qualitatifs, complétés par l'analyse de données quantitatives préexistantes et de la littérature grise.

Où trouve-t-on ces émotions dans ce contexte ? Par le choix de ma démarche, ce n'est pas lorsque je mène des enquêtes auprès des usagers du canal du Midi in situ, que les émotions se donnent le plus à voir. Du moins, lors de ces enquêtes, je n'ai pas particulièrement eu le réflexe de noter les quelques signes, expressions de visage qui pouvaient être lus (d'autant que cela suppose des interprétations qui pourraient être aisément erronées). Mes échanges avec ces personnes sont généralement restés relativement neutres. Certes, ils sont heureux d'être là, ils sont désolés de découvrir des paysages désertiques là où existaient auparavant de magnifiques alignements, mais cela n'a pas semblé un moment opportun pour s'étendre sur leurs émotions et sentiments. II faut préciser aussi que ce n'était pas non plus l'objectif de ces mini-entretiens. Néanmoins, quelques-uns de mes rendez-vous avec divers acteurs ont révélé plus nettement des émotions sous-jacentes à analyser. La presse, plusieurs sites Internet (tels que Tripadvisor ou Replantonslecanaldumidi.fr), ou encore diverses réunions constituent aussi des sources pour se rendre compte des effets des émotions sur la gestion du projet de restauration du canal du Midi.

Immergée quotidiennement dans mon terrain, cet article doit me permettre de prendre du recul par rapport à des émotions qui peuvent m'affecter aussi bien en tant qu'individu qu'en tant qu'apprentie-chercheuse, et de reconsidérer ce qui relève de mon "cap épistémologique " (Dulaurance, Foli, 2013). Ce sont donc mes doutes quant à la manière de pouvoir parler de ma subjectivité lors de la rédaction de ma thèse, dans un contexte de travail chargé d'émotions, qui m’ont au départ incitée à écrire cet article. Pour Julien Bernard, le travail de catégorisation des émotions nécessite :

Un enchâssement de niveaux d'objectivation, une méthode objectivant les émotions en première personne (celles du chercheur), deuxième personne (celles des enquêtés) et troisième personne (lorsque les enquêtés s'attribuent des émotions dans une situation 
particulière et en fonction de celle-ci), mais aussi éventuellement les marqueurs émotionnels (mots, attitudes) entrant dans des jeux de langage spécifiques. (2015 : 9)

Ce découpage en trois types d'individus affectés par des émotions a été mobilisé et adapté à mon travail d'objectivation, puisqu'il permettait de donner un cadre précis pour appréhender les émotions plurielles du chercheur, des enquêtés qui s'expriment en tant qu'individus et des enquêtés qui s'expriment au nom d'une institution. Ces trois types de personnes structureront cet article. Pour l'individu à la première personne, les émotions questionnent le chercheur dans son travail de terrain, et le " pousse " à s'interroger sur les émotions qui l'affectent. Pour l'individu à la seconde personne, il s'agit de s'interroger sur les émotions attendues face à un site patrimonial et touristique tel que le canal du Midi : il s'agit ici de s'intéresser principalement à deux catégories d'acteurs, à savoir les touristes et les habitants. Enfin, j'évoquerai les émotions avouées des enquêtés, autrement dit à la troisième personne, en me concentrant sur celles relayées par les médias et pouvant relever d'une instrumentalisation des émotions.

\section{Les émotions en première personne : une apprentie-chercheuse face aux émotions des autres}

Mon contrat à durée déterminée de trois ans au sein de la Mission mécénat de VNF, obtenu grâce à une CIFRE passée entre mon laboratoire de recherche, l'Équipe interdisciplinaire de recherches en tourisme (EIREST), I'Agence nationale recherche technologie (ANRT) et VNF, a débuté au mois de mai 2013. Mon intégration au sein de l'équipe à Toulouse n'a quant à elle été effective qu'au mois d'octobre de la même année. La Mission mécénat compte aujourd'hui cinq personnes : la responsable de la mission, un chargé de mission auprès des entreprises, une chargée de mission auprès du grand public, une assistante en alternance, et une doctorante, polyvalente. Rattachée directement à la Direction générale à Paris, cette équipe dispose de bureaux mis à disposition par la Direction territoriale Sud-Ouest de Toulouse, sans dépendre administrativement d'elle. Aussi, en plus de devoir justifier de l'intérêt d'employer une doctorante, alors qu'aucune tâche spécifique ne m'avait été assignée, j'ai été confrontée au manque de légitimité d'un nouveau service, hébergé, dont les missions allaient à l'encontre de la culture héritée d'une conception déçue de l'Étatprovidence mais devant faire face tous les jours à son désengagement. Pour diverses raisons (burn-out, congés maladie, etc.), cris et larmes dans les couloirs ont ponctué ce que je considère comme mon terrain au quotidien. Comme j'ai déjà pu l'expliquer, c'est mon appréhension face à l'expression de ces émotions négatives sur mon terrain au quotidien que j'ai souhaité rédiger un article sur l'impact des émotions dans les projets patrimoniaux. En sachant que j'aurais probablement à justifier de l'utilisation du terme "émotions ", je me suis intéressée à quelques définitions. Certaines ont retenu mon attention, dans la mesure où elles m'ont permise de comprendre comment objectiver mes propres émotions face à celles des autres.

Dans le contexte des études portant sur le patrimoine et le processus de patrimonialisation, le terme "émotion " me paraît largement admis. En effet, les " émotions patrimoniales " (Fabre, 2013) ou "émotions patrimonialisantes" (Heinich, 2009) peuvent faire partie du cadre analytique permettant de mieux comprendre les enjeux autour de la mise en patrimoine, à côté du " travail de l'expertise " (Heinich, 2009). Le terme "émotion » est 
alors très rapidement lié à une "mobilisation " autour d'une véritable " cause ", qui peut concerner le sauvetage d'un édifice menacé ou une meilleure mise en valeur. La dimension politique des émotions rejoint alors celle du patrimoine. En effet, selon Nathalie Heinich, "L'on pourrait même dire que l'émotion est la preuve du patrimoine: si la preuve du pudding, selon un célèbre adage, est qu'on le mange, la preuve du patrimoine serait qu'on en est ému " (2012 : 21). Ce terme d'" émotion patrimoniale " peut donc être tout à fait approprié pour un cas comme le canal du Midi. Mais que signifie-t-il exactement ? D'aucuns peuvent utiliser les termes "émotion ", "humeur", "affect» sans effectuer de différenciation, mais le monde scientifique a bien démontré qu'ils n'étaient pas forcément interchangeables.

Les travaux de Denise Van Dam, Jean Nizet et Michel Streith, lors d'une revue de littérature portant sur les émotions, ont attiré mon attention sur divers auteurs :

\begin{abstract}
Sander et Scherer (2009) conceptualisent l'émotion comme un phénomène à composantes multiples comprenant les évaluations de l'événement déclencheur, appelé aussi " cognitive appraisal " (par exemple, se sentir capable de faire face), le ressenti émotionnel (bonheur, honte, colère), les réactions motrices (sourire, froncer les sourcils...), les réactions du système nerveux autonome (accélération du rythme cardiaque, par exemple) et les tendances à agir (se préparer à fuir...). Cela signifie que les émotions sont considérées comme des réponses à des évaluations d'une situation et comme des moteurs pour l'action et qu'en ce sens, elles jouent un rôle décisif dans les conduites humaines. Les émotions sont donc une composante indispensable de la capacité des individus à évaluer des événements, à prendre des décisions et à entreprendre des actions.
\end{abstract}

Garcia-Prieto et al. (2009) proposent quatre classes d'émotions [...] qui ont la particularité de partager des schémas d'évaluation cognitive et des tendances à l'action : les émotions d'accomplissement ; les émotions d'approche ; les émotions de résignation ; les émotions antagonistes (Van Dam et al., 2012 : 320).

En complément à cette définition, dans un article de réflexions méthodologiques, Julien Rebotier précise qu'il faut prendre : « [...] gare à ne pas réduire les émotions aux affects, i.e. à la réaction, à l'immédiat. Il faut contextualiser et ne pas se perdre dans les sensations corporelles immédiates » (2010: 9). Il me semble donc que ces émotions, observées au quotidien, relèvent plus des affects, sont plus immédiats, et ne donnent pas particulièrement lieu à des actions qui pourraient impacter directement l'objet de mes recherches, voire pas du tout (pas de démission, etc.).

Ces différents auteurs insistent donc sur la nécessité d'analyser l'élément déclencheur des émotions, ici la disparition des platanes du canal du Midi. Les affects que j'ai pu observer étaient, eux, bien plus liés à la gestion du projet lui-même et au sentiment de manque de soutien hiérarchique pour les techniciens et relevant donc peut-être plutôt de problématiques liées à la psychologie au travail. Face aux émotions et aux affects des autres mais aussi face à ses propres questionnements, le chercheur doit donc pouvoir différencier ceux qui n'ont pas de réelles conséquences sur son objet d'étude, tout en reconnaissant qu'ils peuvent influencer sa manière de travailler, de ceux qui impactent en profondeur un projet et/ou modifient le système d'acteurs concerné. 


\section{Émotions à la deuxième personne : émotions patrimoniales, expériences touristiques,} rapport à l'arbre

Dans un numéro spécial portant sur "Émotion/Émotions " de la revue Terrains/Théories parue en 2015, le sociologue Julien Bernard revient sur les problèmes épistémologiques posés par la définition des émotions en sciences sociales. II présente notamment les oppositions entre une vision naturaliste - qui considère que des émotions sont innées et universelles -, et une vision constructiviste - pour laquelle les émotions sont vécues par l'individu, mais " relatives aux sociétés, aux époques, aux groupes » (Bernard, $2015: 3$ ). Si, dans ma thèse, je souhaite à la fois utiliser les apports de théories structuralistes et (peutêtre surtout) interactionnistes, m'intéressant aux réseaux d'acteurs et au rôle joué par le non-humain (Callon, Rip, 1992), pour cet article, ma manière d'aborder les émotions observables sur le canal du Midi s'intègre largement dans une vision bourdieusienne et à sa notion d'habitus, notamment à travers les émotions patrimoniales et le rapport des hommes à l'arbre.

Le canal du Midi a connu un processus de patrimonialisation classique. Dans les années 1970 et 1980 , alors que son activité économique connaissait un fort déclin, deux visions opposées se sont confrontées : la première revendiquant la nécessité de moderniser le canal pour permettre le passage de péniches d'un plus gros gabarit (Freycinet), l'autre tenant d'une vision historique de l'ouvrage. Dans ce contexte, le géographe Robert Marconis (1981) est l'un des premiers à avoir posé la question de la mise en patrimoine, parallèlement au discours qui était en train de se construire autour de l'anniversaire de la mort de Pierre-Paul Riquet, concepteur du canal. L'aboutissement de ce processus est l'inscription du canal du Midi sur la liste du Patrimoine mondial de l'Unesco, dont le dossier de candidature a largement été porté par son gestionnaire, VNF, à travers son service communication (Genieys, Négrier, 2002). Nous avons affaire à un processus de patrimonialisation institutionnel, suivant une démarche dite top down, ou par le haut, soutenue par les plaisanciers mais critiquée par le secteur de transport de marchandises. Il peut aussi être utile de préciser ici que la mise en tourisme du canal du Midi s'est cependant faite grâce à des initiatives privées, dans un premier temps par des Anglo-Saxons, et pas selon une volonté institutionnelle. Ceci nous amène donc à dire que cette émotion patrimoniale est à l'origine plutôt animée par le haut. Mais les représentations de chaque partie prenante, représentants de l'État ou acteurs locaux, peuvent entrer en collision. En effet, comme l'avait déjà démontré une étude menée par les universités de Bordeaux, Toulouse et Montpellier, le canal des Deux-Mers dans son ensemble, et pas uniquement le canal du Midi, est " un patrimoine vécu au quotidien » (Béringuier, Salord, 2013). Les témoignages des élus ou des habitants que j'ai pu recueillir vont dans le même sens: en fonction de son emplacement dans le village traversé, c'était un lieu de rencontre, un lieu pour jouer à la pétanque, pour se promener le dimanche en famille, un lieu où l'on apprenait à nager, où les femmes venaient laver leur linge, etc. II s'agissait d'un espace de vie sociale qui, selon M. Émile Delpy, maire de Paraza, constituait, dans le cas de son propre village, un espace de rencontre qui s'est déplacé après l'abattage des platanes. Aujourd'hui donc, les émotions patrimoniales seraient plutôt revendiquées par le bas. Les commentaires postés par les donateurs, dont l'argent est destiné à financer l'abattage des platanes, la reprise des berges ainsi que la replantation, à la fois sur le site Internet Replantonslecanaldumidi.fr et par courrier, témoignent également d'un fort attachement (parfois aussi d'incompréhension), comme le révèle notamment l'utilisation de déterminants possessifs "mon canal " ou 
"notre canal ", probablement influencés par les slogans du moment tels que "Canal du Midi c'est mon histoire aussi" ou "Notre canal en danger». La communication institutionnelle utilise donc les émotions de résignation qui comprennent la tristesse, la peur, la honte et la culpabilité, pour faire ressurgir les émotions d'accomplissement, parmi lesquelles nous retrouvons la fierté (Van Dam et al., 2012 : 320, d'après les écrits de GarciaPrieto).

Toutes ces représentations d'un patrimoine de proximité influencent les propositions de projets d'aménagement émanant des collectivités souhaitant rendre le site agréable pour leurs habitants. Mais, bien souvent, ces projets se heurtent aux conceptions des représentants de l'État qui craignent une banalisation de l'ouvrage. Ceci entraîne évidemment des conflits, des négociations, qui me paraissent constituer une étape au-delà de celle des émotions.

L'attachement au patrimoine est prégnant, mais l'attachement à l'arbre, ici particulièrement au platane, l'est encore plus, comme peut l'illustrer ce commentaire sur la page dédiée à l'appel aux dons pour la restauration du canal du Midi : "l'arbre est le meilleur ami de I'homme: là où il pousse, l'homme s'installe; là où il meurt, l'homme s'enfuit " ${ }^{2}$. En retraçant le rapport de I'homme à l'arbre depuis l'Antiquité, à travers l'œuvre d'auteurs célèbres et selon une vision occidentale, l'historien Alain Corbin nous donne quelques pistes pour comprendre certaines réactions observables sur le canal du Midi. II répète à de nombreuses reprises combien "L'arbre sidère " (Corbin, 2013). Il peut à la fois être l'objet d'une forte admiration, mais aussi d'une crainte. Un parallèle intéressant avec le canal du Midi est à faire lorsque l'auteur rappelle l'ancienneté de ce qui lie l'imaginaire de la cathédrale à celui de l'arbre. J'ai, par exemple, pu constater que le terme "cathédrale » était utilisé pour nourrir la réflexion d'artistes et de designers devant travailler sur divers objets destinés aux grands mécènes du canal.

L'historienne Andrée Corvol résume ainsi les affects liés à l'arbre :

L'arbre était présent quand on naissait, il l'était toujours quand on mourait ; il paraissait échapper à la fuite des années. Au reste, pendant longtemps, les gens auraient été en peine d'en dire l'âge; ils l'estimaient d'après les souvenirs des vieilles personnes ou d'après les chroniques qui le mentionnaient à propos d'un événement. L'arbre âgé était d'autant plus aimé qu'il était plus grand et plus fort. De l'affection à l'adoration, il n'y a qu'un pas. Un tel arbre ne mériterait-il pas d'être divinisé ? (2009 : 11).

Le rôle des émotions face à l'arbre sur un site patrimonial a déjà pu être étudié : Véronique Dassié, docteure à l'EHESS ayant réalisé une thèse d'ethnologie sur les objets d'affection dans l'espace domestique contemporain, sous la direction de Daniel Fabre, s'est intéressée au cas du parc de Versailles après la tempête de 1999. Elle évoque notamment la forte médiatisation de l'atteinte au patrimoine par rapport à la perte économique et humaine :

Les traces du passé, destinées aux générations futures, ont été touchées et leur destruction, en quelques heures, semble produire la stupeur. De Notre-Dame au Panthéon, les plus grands monuments parisiens ont effectivement subi des dégâts. Nous constatons pourtant un relatif désintérêt à l'égard du bâti, oublié lui aussi derrière le constat de la mise à mal d'un autre pôle patrimonial : le végétal. Dans la presse écrite,

\footnotetext{
${ }^{2}$ replantonslecanaldumidi.fr/fr/commentaires?page=6, consulté le 25 avril 2016, commentaire posté le 29 décembre 2015
} 
les monuments cèdent largement la place aux arbres, érigés en victimes de la tempête, identifiée comme une « catastrophe naturelle. (Dassié, $2002: 2$ ).

Un parallèle direct peut être effectué avec le canal du Midi grâce à un autre usager du site Replantonslecanaldumidi.fr: "Ravie de pouvoir aider le canal du midi comme j'ai aidé le parc de Versailles après les grosses tempêtes. C'est plus qu'un patrimoine ; c'est un chemin d'eau qui me ressource $\|^{3}$. II n'est d'ailleurs pas rare d'entendre des suggestions de reproduire l'opération "Adoptez un arbre " sur le canal du Midi. II me semble que les émotions qui se sont exprimées pour Versailles sont issues d'un même héritage sociétal que celles qui s'expriment pour le canal du Midi. Toutefois, pour la restauration de cet ouvrage hydraulique, on ne retrouve pas un élan de solidarité tel que décrit par Véronique Dassié. C'est une analyse qui reste à approfondir, mais il est possible de donner déjà quelques explications : VNF est mal identifié comme interlocuteur privilégié, les donateurs ont moins la possibilité de se sentir physiquement liés à la restauration puisque leurs dons sont perdus entre l'abattage, la reprise des berges et la replantation. Néanmoins, il faut aussi noter que, comme pour le parc de Versailles, l'arbre recentre toutes les attentions et, même si cela évolue, les quelques exemples de sauvetages de chauves-souris ou d'oiseaux (qui révèlent les risques encourus par la faune et la flore présentes sur le canal) trouvent moins d'échos.

Sur un tel site patrimonial, vécu comme un patrimoine du quotidien et où l'arbre est un symbole prégnant, il serait finalement bien plus étonnant de n'y rencontrer aucune émotion et aucun conflit. Les émotions évoquées dans cette seconde partie peuvent s'apparenter aux "émotions d'éblouissement, d'émerveillement et de tendresse " selon la proposition de Denise Van Dam et al (2012). En effet, elles peuvent relever d'une véritable passion ou admiration pour l'ouvrage et son concepteur. Interroger les émotions éprouvées par les usagers locaux du canal du Midi ne peut se faire sans prendre en compte les élus, qui relèvent d'autres catégories, et à l'instrumentalisation politique et médiatique qui en découle.

\section{Émotions à la troisième personne : instrumentalisation des émotions avouées ?}

Comme précisé précédemment, ma position d'apprentie-chercheuse au sein de l'Établissement public administratif VNF, gestionnaire de l'ouvrage, m'a imprégnée de toutes sortes d'affects et ceci m'a paru, à un moment donné, central pour la compréhension de certains tenants et aboutissants dans la gestion du projet de restauration du canal du Midi. Tout le monde connaît la carte postale du canal du Midi (Figure 2), cette voûte verte apparentée à une cathédrale, et les réactions face à sa disparition progressive peuvent être associées aux diverses étapes du deuil définies par la psychologue Élizabeth Kübler-Ross: déni, colère, marchandage, dépression, acceptation. Dès le début de ma thèse, cette image du deuil m'a été présentée par deux membres du personnel de VNF, I'un chef de la subdivision Languedoc-Ouest, basé à Carcassonne, l'autre directrice de cabinet du directeur général, basé à Paris. Tous les deux n'ont donc pas la même approche du terrain, mais ils avaient la même vision du cycle émotionnel que l'on pouvait rencontrer autour du sujet " canal du Midi ». II importe donc de présenter comment cette image trouve un écho particulier à travers la catégorie des acteurs des collectivités territoriales.

\footnotetext{
3 Replantonslecanaldumidi.fr/fr/commentaires ?page=21, consulté le 14 septembre 2015, commentaire posté le 2 juillet 2014
} 
Figure $n^{\circ} 2$ : Le canal du Midi, à proximité du seuil de Naurouze (Aude)

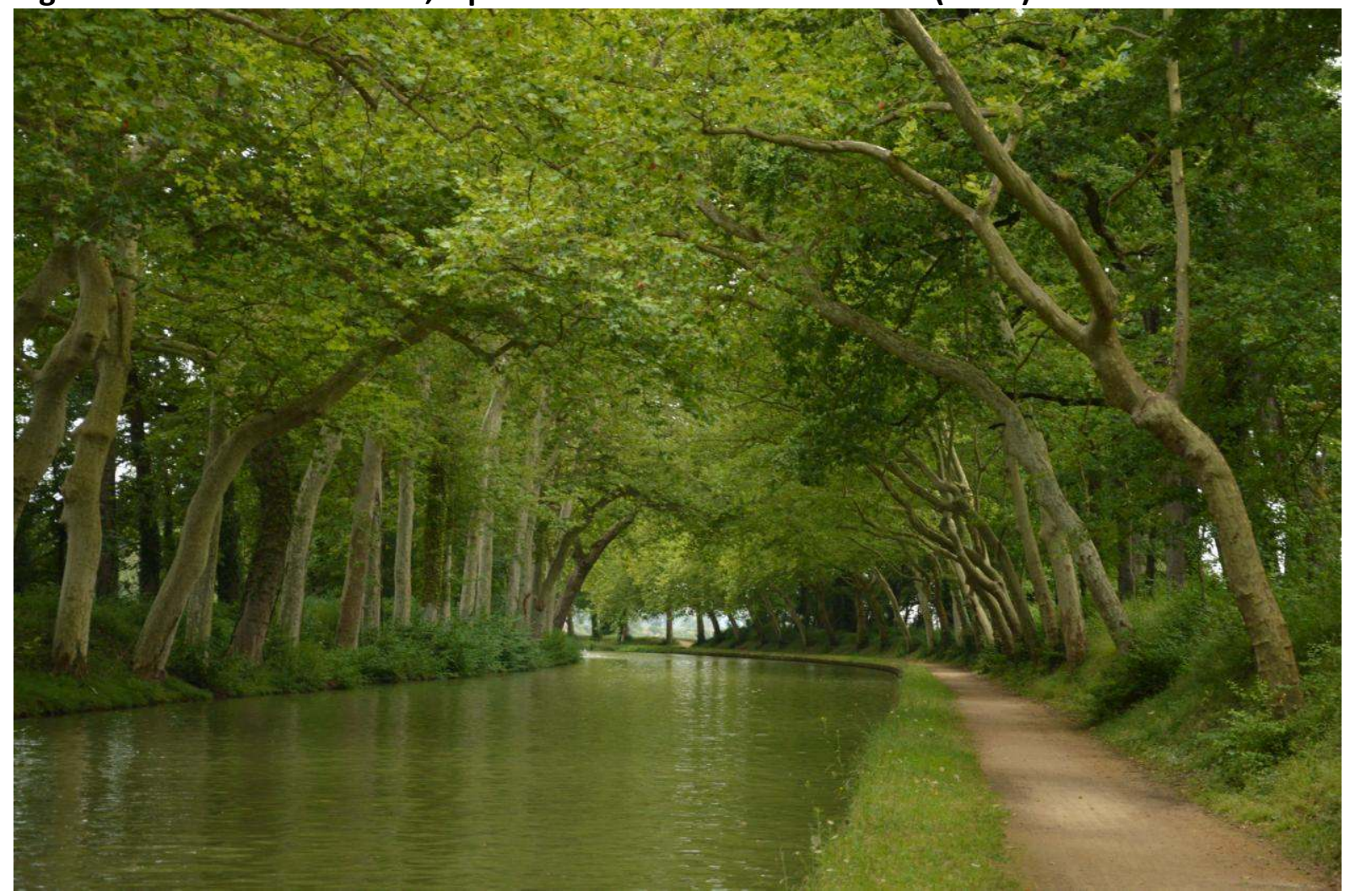

Source : photographie de l'auteure, août 2013

"Le platane : "massacre à la tronçonneuse" sur le canal du Midi " (Garric Audrey, Le Monde, 11 août 2015), "Scandaleux!" (L'Indépendant, 20 août 2015) : ces deux titres d'articles de presse parus à l'été 2015 nous paraissent particulièrement révélateurs des passions, ici plutôt relatives à la colère, susceptibles de graviter autour d'un site comme le canal du Midi. Depuis la découverte en 2006 d'un foyer de chancre coloré sur les platanes situés le long du canal sur la commune de Villedubert (Aude, Languedoc-Roussillon), avec une intensification ces deux dernières années, il est relativement aisé de retrouver des articles de presse se faisant les porte-paroles de la frustration, de la déception, de la colère de citoyens et plus particulièrement d'élus. L'article du Monde entre particulièrement dans le jeu du pathos exprimé par l'un des maires, régulièrement médiatisé sur le sujet depuis les campagnes municipales de 2014 : "Les gens d'ici ont un attachement sentimental au canal du Midi. C'est le point de rencontre des habitants et le centre de l'activité économique du village " (extrait des propos de M. Christian Lapalu, maire de Ventenac-Minervois, cité dans l'article). Ce lien émotionnel au lieu est également partagé par un habitant du village cité dans le même article : "J'ai vu des vieux pleurer la première fois qu'ils ont arraché nos arbres ". Le terme de " massacre ", fortement connoté, est, quant à lui, très régulièrement repris par la presse, essentiellement régionale, à chaque annonce de reprise des actions d'abattage des platanes atteints par la maladie du chancre coloré. De nombreux titres d'articles de presse locale sont emblématiques de cette mobilisation du vocabulaire émotionnelle : "Canal du Midi : le "massacre" continue, 4000 platanes abattus en 2014 » (Midi Libre, 22 janvier 2014), "Carcassonne. Platanes : arrêtez le massacre " (La Dépêche du Midi, 24 janvier 2014), etc. Dans l'article de L'Indépendant cité plus haut, c'est l'anaphore " scandaleux " qui est utilisée pour retranscrire la colère, relevant des émotions antagonistes (Van Dam et al., 2012 : 320, d'après les écrits de Garcia-Prieto), du maire de Sallèles-d'Aude Yves Bastié, qui se bat pour 
l'expérimentation d'un traitement contre le chancre coloré sur les platanes du canal du Midi depuis 2013. Ainsi, colère, frustration, chagrin, exaspération, etc., sont autant d'émotions mobilisées pour dire ce que les bouleversements paysagers du canal du Midi produisent pour les acteurs locaux.

"Je suis prêt à pardonner ": en entendant ces propos de ce même maire de Ventenac Minervois, il m'a semblé qu'on atteignait un point particulier du processus émotionnel. L'élu qui a prononcé cette phrase lors d'une entrevue en bilatéral, et alors qu'il était en campagne pour les départementales 2015, évoquait en fait une réunion à la Chambre de commerce et de l'industrie ( $\mathrm{CCl}$ ) Narbonne datant de novembre 2014 durant laquelle il avait invectivé l'un de mes collègues qui présentait la campagne d'appels aux dons. Cette joute verbale avait été relayée par la presse quotidienne régionale, qui avait rapporté ses propos: "Vous avez replanté des arbres qui, faute de soins, sont tous morts! Allez voir sur Saint-Nazaire ! II n'y a pas eu de concertation et l'abattage systématique signe la mort de nos cœurs de villages ! ". Lors de notre entrevue en tête-à-tête, l'élu s'est dit plus compréhensif et reconnaissait avoir été peut-être trop virulent par le passé. II était également intéressé par ma position au sein de la Mission mécénat, alors qu'il avait une nouvelle idée en tête : celle de proposer de reverser $1 €$ au canal par bouteille vendue par la coopérative de son village, dont il a fait I'annonce dans la presse le 14 août 2015, sans concertation au préalable avec la Mission mécénat, unique entité autorisée à recevoir des dons pour le canal du Midi. Il sera important de voir, à moyen et long termes, quels seront les effets de cette instrumentalisation politique et médiatique. Dans ce cas précis, j'interprète les propos qu'il a eus dans notre entrevue comme une volonté de modifier l'opinion négative que j'aurais pu avoir à son égard, puisqu'il savait que je faisais partie de la Mission mécénat : tout comme la peur est mobilisée à des fins politiques à toutes les échelles (Boucheron, Robin, Payre, 2015), toute la gamme des émotions traverse donc les relations entre les différents acteurs d'un projet patrimonial. Ce récit permet donc de toucher à la nature politique des émotions. En 2014, les conséquences de l'utilisation des émotions ont même été directement visibles sur la gestion du projet de restauration du canal du Midi.

Émotion réelle ou instrumentalisation politique ? Quoi qu'il en soit, l'utilisation des émotions a bel et bien des effets sur l'avenir du projet de restauration. Un moment crucial a notamment eu lieu au printemps 2014 durant les campagnes municipales, moment qui a été qualifié de "fronde des maires de l'Aude ". Afin de tenter de ralentir la propagation de la maladie, VNF faisait appliquer à ses maîtres d'œuvre un principe de précaution, dit de prophylaxie, qui consistait à abattre des arbres visuellement sains de part et d'autre des arbres morts. Après l'annonce de l'abattage de 4000 platanes en janvier 2014 et les élections municipales qui approchaient, plusieurs maires audois se sont révoltés face à cet abattage massif qui leur paraissait déraisonné (émotions antagonistes). Après quelques temps de polémiques, le directeur général de VNF, Marc Papinutti, a accepté de revenir sur le principe prophylactique et ainsi de ne plus abattre d'arbres visuellement sains en avril 2014: "Cette décision temporaire a été prise après que de nombreux maires des départements de l'Aude et de l'Hérault se sont émus, depuis janvier 2014, de l'arrachage systématique des arbres plantés dans la zone dites de "prophylaxie" située 50 mètres en amont et 50 mètres en aval des platanes atteints par le chancre coloré " (Ladepeche.fr, 11 avril 2014). L'avancée du projet de restauration s'en trouve par conséquent aussi ralenti. Les émotions participent ainsi de conflits environnementaux, en tant qu'elles s'imbriquent dans le jeu d'acteurs, accélérant ou ralentissant des projets patrimoniaux et/ou d'aménagement. 
Du point de vue de VNF et des services de l'État (points de vue recueillis lors de plusieurs réunions visant à faire le point sur la mise en œuvre du Cahier de référence pour la replantation du canal du Midi), le fait d'abattre sur de plus petits secteurs pose problème : outre le surcoût financier, facilement réfutable face à l'importance de la vie d'un arbre, les berges sont encore davantage fragilisées par les venues successives des engins massifs nécessaires pour l'abattage des arbres en toute sécurité; de plus, la replantation de séquences paysagères, définies par des paysagistes après un diagnostic de chaque bief, est rendue bien plus difficile puisqu'il faut pouvoir disposer de grandes étendues; enfin, sans que cela ait pu être vérifié, la propagation de la maladie pourrait même être favorisée, puisque les arbres visuellement sains de part et d'autre d'un arbre mort s'avéraient souvent être déjà atteints par la maladie lorsqu'ils étaient coupés (le principe prophylactique visait à contenir la maladie).

\section{Conclusion}

Mon objectif premier à travers cet article était donc de revenir sur des événements pensés comme pouvant m'affecter à un moment donné et de parvenir à les conceptualiser dans une pensée scientifique. Grâce à ce travail, j'ai pu identifier ce que je considérais comme des émotions observables sur mon terrain au quotidien comme relevant plutôt des affects de l'immédiat, n'ayant pas forcément d'impact en termes d'action et n'ayant donc pas toujours d'effets sur les projets étudiés plus en profondeur dans ma thèse. II s'agit donc d'un premier pas vers une objectivation de mes travaux de recherche, tout en sachant que je ne pourrai pas nier une subjectivité qui ne pourra que subsister.

J'ai voulu rappeler que, face à un patrimoine faisant l'objet d'expériences touristiques, fortement lié à la thématique de l'espace vécu, et dont la figure de l'arbre est un symbole prégnant, une approche par les émotions est classique. On peut rapprocher ces émotions de celles dites "d'accomplissement", complétées par les émotions sensorielles comprenant l'éblouissement, l'émerveillement et la tendresse, sur une proposition de D. Van Dam et al. (2012 : 320 et 326). Mais il apparaît que les émotions peuvent aussi révéler la complexité des relations entre acteurs, ce qui est intéressant lorsqu'on adopte une approche systémique. En effet, pour un cas comme le canal du Midi, elles sont le reflet des désaccords entre les différents acteurs publics, notamment les représentants de l'État (VNF, services déconcentrés) et les collectivités territoriales (les élus des communes dites « mouillées par le canal du Midi »). On peut donc considérer l'abattage des platanes comme un élément déclencheur d'émotions qui peuvent être qualifiées d' " antagonistes » (toujours d'après la classification reprise par Van Dam et al., 2012 : 320), qui comprend la colère et qui a été motrice pour une action bien précise : I'abandon du principe de prophylaxie.

Rédiger cet article m'a montré que, finalement, mon appréhension face à des émotions de tristesse et de colère était peut-être moins justifiée qu'une appréhension face à des moments joyeux et des liens d'amitié qui se sont tissés, tout autant susceptibles de remettre en cause mon objectivité. 


\section{Bibliographie}

BÉRINGUIER P., SALORD T. (2013), "Canal du Midi, un patrimoine vécu au quotidien », MidiPyrénées patrimoine, $\mathrm{n}^{\circ} 34$, pp. 65-68.

BERNARD J. (2015b), "Les voies d'approche des émotions. Enjeu de définition et catégorisations ", Terrains/Théories, $n^{\circ} 2$, en ligne : http://teth.revues.org/196

BOUCHERON P., ROBIN C., PAYRE R. (2015), L'Exercice de la peur. Usages politiques d'une émotion, Lyon, Presses universitaires de Lyon, Collection Grands débats : mode d'emploi, $84 \mathrm{p}$.

CALLON M., RIP A. (1992), " Humains, non-humains : morale d'une coexistence ", in Theys J., Kalaora B. (dir.), La Terre outragée, Paris, Autrement, pp. 140-156.

CORBIN A. (2013), La Douceur de l'ombre. L'arbre, source d'émotions, de l'Antiquité à nos jours, Paris, Fayard, $364 \mathrm{p}$.

CORVOL A. (2009), L'Arbre en Occident, Paris, Fayard, 370 p.

DASSIÉ V. (2002), "Décembre 1999, tempête sur Versailles. Chronique d'une émotion ", Ethnologies comparées, 8 p., en ligne : http://alor.univ-montp3.fr/cerce/r4/v.d.htm.<halshs$\underline{00506020}$

DULAURANCE M., FOLI O. (2013), «Tenir le cap épistémologique : ajustements nécessaires et connaissances produites en contexte $"$, Études de communication, $n^{\circ} 40, p p .59-76$, en ligne : https://edc.revues.org/5118

FABRE D. (2013) (dir.), Émotions patrimoniales, Paris, Maison des sciences de l'homme, collection Cahiers d'ethnologie de la France, $410 \mathrm{p}$.

GARCIA-PRIETO G., TRAN V. et WRANIK T. (2009) Les théories de l'évaluation cognitive et de la différenciation des émotions : une clé pour comprendre le vécu émotionnel au travail, in DELOBBE N. et al. (Eds), Comportement organisationnel, Bruxelles, De Boeck, pp. 195-222.

GENIEYS W., NÉGRIER E. (2002), « Le canal du Midi : du patrimoine mondial à son impossible développement culturel inter-régional », in Négrier E. (dir.), Patrimoine culturel et décentralisation. Une étude en Languedoc-Roussillon, Paris, L'Harmattan, pp. 199-228.

GUMUCHIAN H., GRASSET E., LAJARGE R., ROUX E. (2003), Les Acteurs, ces oubliés du territoire, Paris, Anthropos, $186 \mathrm{p}$.

HEINICH N. (2012), "Les émotions patrimoniales: de l'affect à l'axiologie ", Social Anthropology, $n^{\circ} 20 / 2012$, pp. 19-33.

HEINICH N. (2009), La Fabrique du patrimoine. De la cathédrale à la petite cuillère, Paris, Éditions de la Maison des sciences de l'homme, collection Ethnologie de la France, 288 p.

MARCONIS R. (1981), "Les canaux du Midi, outil économique ou monument du patrimoine régional ? ", Revue géographique des Pyrénées et du Sud-Ouest, vol. 52, n¹/1981, pp. 7-40, en ligne : http://www.persee.fr/doc/rgpso_0035-3221_1981_num_52_1_3638

SANDER D. et SCHERER K. (2009) Traité de psychologie des émotions, Paris, Dunod.

REBOTIER J. (2010), "Géographie et émotions », Réflexions méthodologiques, n¹, en ligne: www.labovespa.ca/IMG/pdf/reflexionmethono1 mai2010.pdf

VIOLIER P. (2003), "Les acteurs du tourisme ", in Stock M. (dir.), Le Tourisme. Acteurs, lieux et enjeux, Paris, Belin, collection Belin Sup Géographie, pp. 167-208.

VAN DAM D. et al (2012) "Les émotions comme lien entre l'action collective et l'activité professionnelle: le cas de l'agriculture biologique ", Nature, Sciences Sociétés, vol. 20, $n^{\circ} 3 / 2012$, pp. 318-329, en ligne: http://www.nssjournal.org/articles/nss/pdf/2012/03/nss120026.pdf

VOIES NAVIGABLES DE FRANCE, 2015, "Le Programme de replantation ", Le Canal des deux Mers en bateau, à pied, à vélo, carte du réseau 2015. 